\title{
變糹面の部材を有する架棈の挫屈に就いて（其の 2)
}

\author{
正會員 蛭 田 捨 太 郎*
}

\section{內 容 梗 概}

柱材の斷面が漸變する場合，柱脚固定の架構の挫屈荷重を Bessel 函數に依り求めたもので 岕る。

目次

\section{$\S 1$ 緒 : 言 \\ $\S 2$ 基礎微分方程式及びその解 \\ $\S 3 \mathrm{~m}=0$ なる場合の解 \\ $\S 4 \mathrm{~m}=1$ なる場合の解}

\section{$\$ 1$ 緒 言}

岡節架構の挫屈の研究は相當古くから行われてい る。然く乍ら柱材の斷面が漸變する場合に就ての研究 は殆どその報告を見ない。

筆者は柱材の斷面が $\mathrm{I}(\mathrm{x})=\mathrm{I}_{0}\left(\frac{\mathrm{x}}{\mathrm{a}}\right)^{\mathrm{m}}(\mathrm{m}=0,1,2$, 3，4）の如く潮變与る場合に就てさきに柱脚鉸の場合 に就て報告**したが，本報に於ては柱脚固定を取披 5。双挫屈理論の基礎微分方程式は從來の二階微分方 程式では不充分であることは既に指摘せられ

$$
E I \frac{d^{4} y}{d x^{4}}+P \frac{d^{2} y}{d x^{2}}=0
$$

に依るべきであると言われている。

又漸變糹面漸變軸壓に對 しては, 高橋利衞博士が (1.1) 式を擴張して

$$
\frac{d^{2}}{d x^{2}}\left[\operatorname{EI}(x) \frac{d^{2} y}{d x^{2}}\right]+\frac{d}{d x}\left[P(x) \frac{d y}{d x}\right]=0
$$

を誘導している。この一般解は Cowley-Levy の方法 により無限級數を用いて求めるのであるが，柱材の如 き單一材の挫屈荷重を決定する場合と雖も，相當複雜 であり, 更に本研究の如く架構の一部材が變斷面の時 は可成の困難を伴なう。

そこで箯者は從來の一階の微分方程式を採用して, 最小挫屈荷重を得る挫屈の型を想定して解を求めたる のである。，斷面一定の場合の從來の解は本研究に於る $\mathrm{m}=0$ の場合であり, 文, 長柱の解は梁の剛度を特別 な值にすれば求め得る。

\section{§2 基礎微分方程引及びその解}

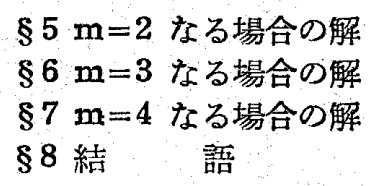

$\S 5 \mathrm{~m}=2$ なる場合の解 $\S 6 \mathrm{~m}=3$ なる場合の解 $\S 7 \mathrm{~m}=4$ なる場合の解 $\S 8$ 結一語

第1 圆に示与如き架構に於て P が挫屈荷重 $\mathrm{P}_{\mathrm{cr}} に$ 近づけば，架構は不安定狀態になり $\mathrm{P}$ が $\mathrm{P}_{\mathrm{cr}}$ を越え た瞬間に於て次の安定狀態に移行する。この时の挫屈 型を點線にて示す如く假定すれば釣合の式は原點を假 想の點 $\bigotimes に と り$ 横軸に $\mathrm{y}$, 縱軸に $\mathrm{x}$ を採れば

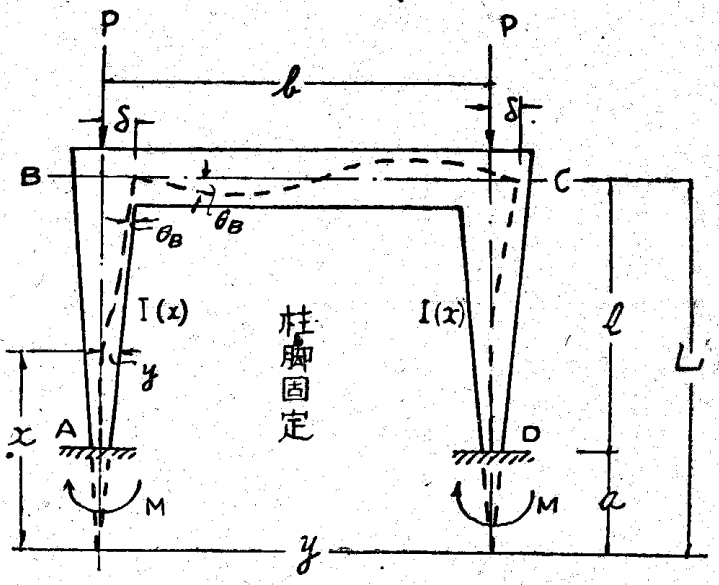

$\otimes$

$$
\begin{array}{r}
\mathrm{EI}(\mathrm{x}) \frac{\mathrm{d}^{2} \mathrm{y}}{\mathrm{dx}^{2}}+\mathrm{P}(\mathrm{y}-\delta)+\mathrm{M}=0 \cdots \cdots(2.1) \\
\mathrm{I}(\mathrm{x})=\mathrm{I}_{0}\left(\frac{\mathrm{x}}{\mathrm{a}}\right)^{\mathrm{m}} \text { を 上式に代入し少しく書換へれば } \\
\mathrm{x}^{-\mathrm{n}} \frac{\mathrm{d}^{2} \mathrm{y}}{\mathrm{dx}^{2}}+\mathrm{k}^{2} \mathrm{y}=\mathrm{k}^{2}(\delta-\theta) \cdots \cdots \cdots \cdots(2.2)
\end{array}
$$

を得る。妶に $\mathrm{n}=-\mathrm{m}, \mathrm{k}=\left(\frac{\mathrm{Pa}^{\mathrm{m}}}{\mathrm{EI}_{0}}\right)^{\frac{1}{2}}, \theta=\frac{\mathrm{P}}{\mathrm{M}}$ である。 (2.2) は Riccati の微分方程式に變形出來るから， その一般解は次のや5に Bessel 函數を用いて表せ る。

* 早稻田大學理工學部講師

** 蛙时捨太郎：變斷面の部材を有する架構の挫屈に就いて 建築學會論文集 第 36 號 昭和 22 年 12 月 


$$
\mathrm{y}=\mathrm{x}^{\frac{1}{2}} \mathrm{C}_{1} \mathrm{~J}_{\nu}(\mathrm{z})+\mathrm{x}^{\frac{1}{2}} \mathrm{C}_{2} \mathrm{Y}_{\nu}(\mathrm{z})+\delta-\theta \cdots(2.3)
$$

妶に

$$
\nu=\frac{1}{n+2}, \quad z=\frac{2 k}{n+2} x^{\frac{n+2}{2}}
$$

$\mathrm{C}_{1}, \mathrm{C}_{2}$ は積分常數, $\mathrm{J}_{\nu}(\mathrm{z}), \mathrm{Y}_{\nu}(\mathrm{z})$ は夫々第 $\nu$ 位の - 第一種, 第二種の Bessel 函數である。

(2.3) \$5

$$
\begin{gathered}
\frac{\mathrm{dy}}{\mathrm{dx}}=\mathrm{C}_{1}\left\{\frac{1}{\sqrt{\mathrm{x}}} \mathrm{J}_{\nu}(z)-\mathrm{kx}^{\frac{\mathrm{n}+1}{2}} \mathrm{~J}_{v+1}(z)\right\} \\
+\mathrm{C}_{2}\left\{\frac{1}{\sqrt{\mathrm{x}}} \mathrm{Y}_{\nu}(z)-\mathrm{kx}^{\frac{\mathrm{n}+1}{2}} \mathrm{Y}_{v+1}(z)\right\}
\end{gathered}
$$

柱脚固定としての境界條件は

$$
\mathrm{y}=0, \frac{\mathrm{dy}}{\mathrm{dx}}=0
$$$$
\text { 固定端に於て } \mathrm{x}=\mathrm{a} \text { の時 }
$$

上端 $\mathrm{B}, \mathrm{C}$ に於て $\mathrm{x}=\mathrm{L}$ の時

$$
\mathrm{y}=\delta, \frac{\mathrm{dy}}{\mathrm{dx}}=\frac{\mathrm{Mb}}{6 \mathrm{EI}_{1}}
$$

(2.6)によつて $\mathrm{C}_{1}, \mathrm{C}_{2}$ を計算すると次の通りにな

一。但し $z_{\mathrm{a}}=|z|_{x=a}$,とする。

$$
\mathrm{C}_{1}=\frac{(\delta-\theta)\left\{\mathrm{a}^{-\frac{1}{2}} \mathrm{Y}_{\nu}\left(z_{\mathrm{a}}\right)-\mathrm{ka}^{\frac{\mathrm{n}+1}{2}} \mathrm{Y}_{\nu+1}\left(z_{\mathrm{a}}\right)\right\}}{\mathrm{ka}^{\frac{\mathrm{n}+2}{2}}\left\{\mathrm{~J}_{\nu}\left(\mathrm{z}_{\mathrm{a}}\right) \mathrm{Y}_{\nu+1}\left(\mathrm{z}_{\mathrm{a}}\right)-\mathrm{Y}_{\nu}\left(\mathrm{z}_{\mathrm{a}}\right) \mathrm{J}_{\nu+1}\left(\mathrm{z}_{\mathrm{a}}\right)\right.}
$$$$
\mathrm{C}_{2}=\frac{-(\delta-\theta)\left\{\mathrm{a}^{-\frac{1}{2}} \mathrm{~J}_{\nu}\left(z_{\mathrm{a}}\right)-\mathrm{ka}^{\frac{\mathrm{n}+1}{2}} \mathrm{~J}_{\nu+1}\left(\mathrm{z}_{\mathrm{a}}\right)\right\}}{\mathrm{ka}^{\frac{\mathrm{n}+2}{2}}\left\{\left\{\mathrm{~J}_{v}\left(\mathrm{z}_{\mathrm{a}}\right) \mathrm{Y}_{\nu+1}\left(\mathrm{z}_{\mathrm{a}}\right)-\mathrm{Y}_{v}\left(\mathrm{z}_{\mathrm{a}}\right) \mathrm{Y}_{\nu+1}\left(z_{\mathrm{a}}\right)\right\}\right.}
$$

之等を (2.3) (2.5) に代入して

$$
\begin{aligned}
& \mathrm{y}=\frac{(\delta-\theta)\left\{\mathrm{a}^{-\frac{1}{2}} \mathrm{Y}_{\nu}\left(z_{\mathrm{a}}\right)-\mathrm{ka}^{\frac{\mathrm{n}+1}{2}} \mathrm{Y}_{\nu+1}\left(z_{\mathrm{a}}\right)\right\}\left[\frac{1}{\sqrt{\mathrm{x}}}\right.}{\mathrm{ka}^{\frac{\mathrm{n}+1}{2}}\left\{\mathrm{~J}_{\nu}\left(z_{\mathrm{a}}\right)\right.} \\
& \mathrm{J}_{\nu}(z)-(\delta-\theta)\left\{\mathrm{a}^{-\frac{1}{2}} \mathrm{~J}_{\nu}\left(\mathrm{z}_{\mathrm{a}}\right)-\mathrm{ka} \mathrm{a}^{\frac{\mathrm{n}+1}{2}} \mathrm{~J}_{v+1}\left(\mathrm{z}_{\mathrm{a}}\right)\right. \\
& \mathrm{Y}_{v+1}\left(\mathrm{Z}_{\mathrm{a}}\right)-\mathrm{Y}_{\nu}\left(\mathrm{Z}_{\mathrm{a}}\right) \mathrm{J}_{\nu+1}\left(\mathrm{Z}_{\mathrm{a}}\right) \\
& \left.\int \mathrm{x}^{\frac{1}{2}} \mathrm{Y}_{\nu}(\mathrm{z})\right]+\delta-\theta
\end{aligned}
$$

$$
\frac{\mathrm{dy}}{\mathrm{dx}}=\frac{(\delta-\theta)\left[\left\{\mathrm{a}^{-\frac{1}{2}} \mathrm{Y}_{\nu}\left(z_{\mathrm{a}}\right)-\mathrm{ka}^{\frac{\mathrm{n}+1}{2}} \mathrm{Y}_{\nu+1}{ }^{2}\left(z_{\mathrm{a}}\right)\right\}\right.}{\mathrm{ka}^{\frac{\mathrm{n}+1}{2}}\left\{\mathrm{~J}_{\nu}\right.}
$$

$$
\begin{aligned}
& \left\{\frac{1}{\sqrt{\mathrm{X}}} \mathrm{J}_{v}(\mathrm{z})-\mathrm{kx}^{\frac{\mathrm{n}+1}{2}} \mathrm{~J}_{v+1}(\mathrm{z})\right\}-\left\{\mathrm{a}^{-\frac{1}{2}} \mathrm{~J}_{v}\right. \\
& \left(\mathrm{z}_{\mathrm{a}}\right) \mathrm{Y}_{v+1}\left(\mathrm{z}_{\mathrm{a}}\right)-\mathrm{Y}_{\nu}\left(\mathrm{z}_{\mathrm{a}}\right) \mathrm{J}_{v+1}\left(z_{\mathrm{a}}\right) \\
& \left.\left(\mathrm{z}_{\mathrm{a}}\right)-\mathrm{ka}^{\frac{\mathrm{n}+1}{2}} \mathrm{~J}_{v+1}\left(\mathrm{z}_{\mathrm{a}}\right)\right\}\left\{\frac{1}{\sqrt{\mathrm{x}}} \mathrm{Y}_{\nu}(\mathrm{z})-\right. \\
& \left.\mathrm{kx}^{\frac{\mathrm{n}+1}{2}} \mathrm{Y}_{v+1}(\mathrm{z})\right\}
\end{aligned}
$$

(2.9), (2.10)を(2.7) に代入して

$$
\begin{aligned}
& \mathrm{a}^{-\frac{1}{2}} \mathrm{~L}^{-\frac{1}{2}} \varphi-\mathrm{ka}^{\frac{\mathrm{n}+1}{2}} \mathrm{~L}^{-\frac{1}{2}} \psi-\mathrm{ka}^{-\frac{1}{2}} \mathrm{~L}^{\frac{\mathrm{n}+1}{2}} \xi+\mathrm{k}^{2} \mathrm{a} \\
& \left.\frac{\mathrm{n+1}}{2} \mathrm{~L}^{\frac{\mathrm{n}+1}{2}} \eta\right) \delta-\left(\mathrm{a}^{-\frac{1}{2}} \mathrm{~L}^{-\frac{1}{2}} \varphi-\mathrm{ka}^{\frac{\mathrm{n}+1}{2}} \mathrm{~L}^{-\frac{1}{2}} \psi-\right. \\
& \mathrm{ka}^{-\frac{1}{2}} \mathrm{~L}^{\frac{\mathrm{n}+1}{2}} \xi+\mathrm{k}^{2} \mathrm{a}^{\frac{\mathrm{n}+1}{2}} \eta+\mathrm{kwa}^{\frac{\mathrm{n}+2}{2}} \text { D) } \theta=0
\end{aligned}
$$$$
\left(\mathrm{L}^{\frac{1}{2}} \mathrm{a}^{-\frac{1}{2}} \varphi-\mathrm{ka}^{\frac{\mathrm{n}+1}{2}} \mathrm{~L}^{\frac{1}{2}} \psi\right) \delta-\left(\mathrm{ka}^{\frac{\mathrm{n}+2}{2}} \mathrm{D}+\mathrm{L}^{\frac{1}{2}}\right.
$$$$
\left.\mathrm{a}^{-\frac{1}{2}} \varphi-\mathrm{ka}^{\frac{\mathrm{n}+1}{2}} \mathrm{~L}^{\frac{1}{2}} \psi\right) \theta=0 \cdots \ldots \ldots \ldots(2.12)
$$

こっで $\mathrm{P}$ が $\mathrm{P}_{\mathrm{cr}}$ を少しく越えた場合を考えるので あるから $\theta, \delta$ は同時に零となることはない。從つて (2.11)，(2.12) に於る $\theta, \delta$ の係數にて作られる行 列式を $\Delta$ とすれば

$$
\Delta=0
$$

上式を展開し整理すれば

$$
\begin{gathered}
\mathrm{ka}^{\frac{\mathrm{n}+2}{2}} \mathrm{D}\left\{\left(\omega \mathrm{L}^{\frac{1}{2}} \mathrm{a}^{-\frac{1}{2}}-\mathrm{a}^{-\frac{1}{2}} \mathrm{~L}^{-\frac{1}{2}}\right) \varphi-\mathrm{ka}^{\frac{\mathrm{n}+1}{2}}\right. \\
\left(\omega \mathrm{L}^{\frac{1}{2}}-\mathrm{L}^{-\frac{1}{2}}\right) \psi+\mathrm{ka}^{-\frac{1}{2}} \mathrm{~L}^{\frac{\mathrm{n}+1}{2}} \xi-\mathrm{k}^{2} \mathrm{~L}^{\frac{\mathrm{n}+1}{2}} \\
\left.\mathrm{a}^{\frac{\mathrm{n}+1}{2}} \eta\right)=0
\end{gathered}
$$

妶に $\phi, \psi, \xi, \eta, \mathrm{D}$ 等は下記の通りである。

$\mathrm{D}=\mathrm{J}_{v}\left(\mathrm{Z}_{\mathrm{a}}\right) \mathrm{Y}_{v+1}\left(\mathrm{Z}_{\mathrm{a}}\right)-\mathrm{Y}_{v}\left(\mathrm{Z}_{\mathrm{a}}\right) \mathrm{J}_{v+1}\left(\mathrm{Z}_{\mathrm{a}}\right)$

$\xi=\mathrm{Y}_{\nu}\left(\mathrm{z}_{\mathrm{a}}\right) \mathrm{J}_{\nu+1}\left(\mathrm{z}_{\mathrm{L}}\right)-\mathrm{J}_{\nu}\left(\mathrm{z}_{\mathrm{a}}\right) \mathrm{Y}_{\nu+1}\left(\mathrm{z}_{\mathrm{L}}\right)$

$\varphi=\mathrm{J}_{\nu}\left(\mathrm{z}_{\mathrm{L}}\right) \mathrm{Y}_{\nu}\left(\mathrm{z}_{\mathrm{a}}\right)-\mathrm{J}_{\nu}\left(\mathrm{z}_{\mathrm{a}}\right) \mathrm{Y}_{\nu}\left(\mathrm{z}_{\mathrm{L}}\right)$

$\eta=J_{v+1}\left(z_{\mathrm{L}}\right) \mathrm{Y}_{\nu+1}\left(z_{\mathrm{a}}\right)-\mathrm{J}_{\nu+1}\left(z_{\mathrm{a}}\right)$

$\psi=\mathrm{J}_{\nu}\left(\mathrm{z}_{\mathrm{L}}\right) \mathrm{Y}_{\nu+1}\left(\mathrm{z}_{\mathrm{a}}\right)-\mathrm{Y}_{\nu}\left(\mathrm{z}_{\mathrm{L}}\right) \mathrm{J}_{\nu+1}\left(\mathrm{z}_{\mathrm{a}}\right)$
$\omega=\frac{\mathrm{Pb}}{6 \mathrm{EI}_{1}}$

（2.15）を(2.14)に代入して，この超越方程式を解 けば挫屈荷重が得られる。

\section{$\S 3 \mathrm{~m}=0$ 极る場合の解}

$\mathrm{m}=0, \mathrm{n}=0$ ならば $\nu=\frac{1}{2} \quad z=\mathrm{kx} \quad \mathrm{z}_{\mathrm{L}}=\mathrm{kL}$ $\mathrm{z}_{\mathrm{a}}=\mathrm{ka}$ 
レが半奇數の時は

$$
\begin{aligned}
& J_{p+\frac{1}{2}}(z)=(-1)^{p} \sqrt{\frac{2}{\pi}} z^{p+\frac{1}{2}}\left(\frac{1}{z} \frac{d}{d z}\right)^{p}\left(\frac{\sin z}{z}\right) \\
& Y_{p+\frac{1}{2}}(z)=(-1)^{p+1} \sqrt{\frac{2}{\pi}} z_{p+\frac{1}{2}}\left(\frac{1}{z} \frac{d}{d z}\right)\left(\frac{\cos z}{z}\right)
\end{aligned}
$$

（3.1）（2.15）に代入し，更にその結果を (2.14) に入れると

$$
\begin{aligned}
\tan \mathrm{k} l & =-\frac{\omega}{\mathrm{k}}=-\frac{\mathrm{k} l \mathrm{I}_{0} \mathrm{~b}}{6 \mathrm{I}_{1} l} \\
& =-\frac{\mathrm{kl}}{6 \beta}\left(\text { 妶に } \beta=\frac{\mathrm{I}_{1} \mathrm{l}}{\mathrm{I}_{0} \mathrm{~b}}\right)
\end{aligned}
$$

これが挫屈荷重を決定する式であつてTimoshenko の挫屈理論*に載つている式と一致する。

(3.2) の特別の場合を考慮すると

i) $\mathrm{I}_{1} \rightarrow 0$ のとき $\tan \mathrm{k} l=-\infty \quad \therefore \mathrm{k} l=\frac{\pi}{2}$

$$
\therefore \mathrm{P}_{\mathrm{or}}=\frac{\pi^{2} \mathrm{EI}}{41^{2}}
$$

ii) $\mathrm{I}_{1} \rightarrow \infty$ のとき $\tan \mathrm{k} l=0 \quad \therefore \mathrm{k} l=\pi$

$$
\therefore \mathrm{P}_{\mathrm{cr}}=\frac{\pi^{2} \mathrm{EI}}{1^{2}}
$$

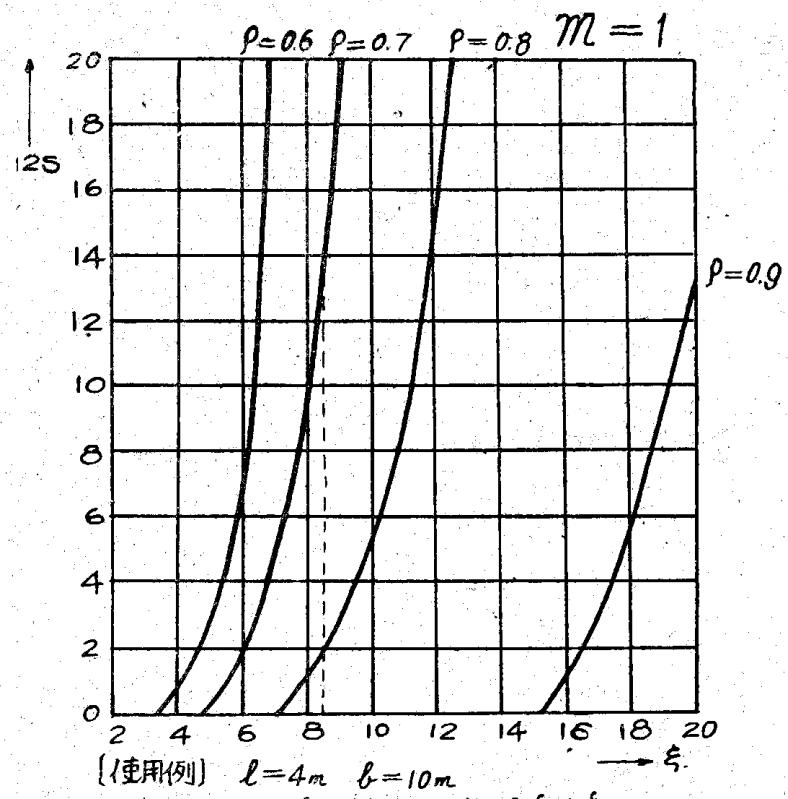

断面 $30 \times 60^{\circ}$ 枆上端 $80^{\circ} \times 40^{\circ}$

$\rho=\sqrt{\frac{4}{8}}=07 \quad 125=\frac{12 a I_{1}}{b I_{0}}=\frac{12 \times 4 \times 0.3 \times 06^{3}}{10 \times 0.4^{4}}=14$

$$
\xi_{1}=8.6
$$$$
\therefore P_{c r}=\xi_{1}^{2} \frac{E_{C} I_{0}}{4 a L}=8.5^{2} \times \frac{2.1 \times 10^{4} \times 0.4^{4}}{4 \times 4 \times 8 \times 12}=31 \mathrm{ton}
$$

第 2 圖
これは Euler の挫屈荷重である。

(3.2) は $\eta_{1}=\tan \mathrm{k} l, \eta_{2}=-\frac{\mathrm{k} l}{6 \beta}$ の最初の交點を求め て品として解く

$$
\mathrm{P}_{\mathrm{cr}}=\xi_{1} \frac{\mathrm{EI}_{0}}{1^{2}}
$$

$\mathrm{m}=0$ ならば $\mathrm{I}=$ const 即斷面一定の場合は本研究 に於る挫屈荷重決定式 (2.14) の特別の場合である。

\section{$\S 4 \mathrm{~m}=1$ なる場合の解}

$$
\begin{gathered}
\mathrm{m}=1, \quad \mathrm{n}=-1 \quad \therefore \nu=1 \quad z=2 \sqrt{\mathrm{x}} \mathrm{k} \\
\mathrm{z}_{\mathrm{L}}=z \sqrt{\mathrm{L} \mathrm{k}=\alpha \mathrm{k},} \quad \mathrm{z}_{\mathrm{a}}=2 \sqrt{\mathrm{a} \mathrm{k}=\beta \mathrm{k}}
\end{gathered}
$$

$$
\text { 娃に } \alpha=2 \sqrt{\mathrm{L}} \quad \beta=2 \sqrt{\mathrm{a}}
$$

依つて (2.15) は

$$
\left.\begin{array}{l}
\varphi=\mathrm{Z}_{1}(\alpha \mathrm{k}) \mathrm{Y}_{1}(\beta \mathrm{k})-\mathrm{J}_{1}(\beta \mathrm{k}) \mathrm{Y}_{1}(\alpha \mathrm{k}), \\
\xi=\mathrm{Y}_{1}(\beta \mathrm{k}) \mathrm{J}_{2}(\alpha \mathrm{k})-\mathrm{J}_{1}(\beta \mathrm{k}) \mathrm{Y}_{2}(\alpha \mathrm{k}) \\
\psi=\mathrm{J}_{1}(\alpha \mathrm{k}) \mathrm{Y}_{2}(\beta \mathrm{k})-\mathrm{Y}_{1}(\alpha \mathrm{k}) \mathrm{J}_{2}(\beta \mathrm{k}), \\
\eta=\mathrm{J}_{2}(\alpha \mathrm{k}) \mathrm{Y}_{2}(\beta \mathrm{k})-\mathrm{J}_{2}(\beta \mathrm{k}) \mathrm{Y}_{2}(\alpha \mathrm{k})
\end{array}\right\}
$$

及

$$
\begin{aligned}
& J_{2}(z)=\frac{2}{z} J_{1}(z)-J_{0}(z), \\
& Y_{2}(z)=\frac{2}{z} Y_{1}(z)-Y_{0}(z)
\end{aligned}
$$

の關係があるから之等を(2.14) に代入する。その結 果

$$
\begin{aligned}
& \frac{\mathrm{J}_{1}(\alpha \mathrm{k})-\mathrm{pY}_{1}(\alpha \mathrm{k})}{\mathrm{J}_{0}(\alpha \mathrm{k})-\mathrm{pY}_{0}(\alpha \mathrm{k})}=\frac{2 \mathrm{k}}{\omega \alpha}=\frac{12 \mathrm{~s}}{\alpha \mathrm{k}} \\
& \text { 始k } \mathrm{p}=\frac{\mathrm{J}_{0}(\beta \mathrm{k})}{\mathrm{Y}_{0}(\beta \mathrm{k})}, \quad \mathrm{s}=\frac{\mathrm{aI}_{1}}{\mathrm{I}_{0}}
\end{aligned}
$$

之に依つて挫屈荷重を定めることが出來る。(4.3)を 解くために

$\alpha \mathrm{k}=\xi, \beta=\rho \alpha(\rho<1)$ と括けば

$$
\frac{\left\{\mathrm{J}_{1}(\xi)-\frac{\mathrm{J}_{0}(\rho \xi)}{\mathrm{Y}_{0}(\rho \xi)} \mathrm{Y}_{1}(\xi)\right\} \xi}{\mathrm{J}_{0}(\xi)-\frac{\mathrm{J}_{0}(\rho \xi)}{\mathrm{Y}_{0}(\rho \xi)} \mathrm{Y}_{0}(\xi)}=12 \mathrm{~s}
$$

(4.4) 式の右邊は常數であるから左邊を $\rho$ を媒介變 數とするほの函數とみなして圖表化すればよい。 圖で求めた根を $\xi_{1}$ とすると

$$
\begin{aligned}
& \mathrm{P}_{\mathrm{cr}}=\xi_{1}{ }^{2} \frac{\mathrm{EI}_{0}}{4 \mathrm{aL}} \\
& \mathrm{I}_{1} \rightarrow 0 \text { の時は } \\
& \mathrm{J}_{1}(\alpha \mathrm{k})-\mathrm{pY}_{1}(\alpha \mathrm{k})=0
\end{aligned}
$$

S. Timoshenko: Theory of Elastic Stability Chapter II Section 28 p. 153-1936. First Edition 
$I_{1} \rightarrow \infty$ の時は

$$
\mathrm{J}_{0}(\alpha \mathrm{k})-\mathrm{pY}_{0}(\alpha \mathrm{k})=0
$$

(4.7) 式につき考えると

$$
\begin{aligned}
& \frac{\mathrm{Y}_{0}(\alpha \mathrm{k})}{\mathrm{Y}_{0}(\beta \mathrm{k})}-\frac{\mathrm{J}_{0}(\alpha \mathrm{k})}{\mathrm{J}_{0}(\beta \mathrm{k})}=0 \quad\left(\because \mathrm{p}=\frac{\mathrm{J}_{0}(\beta \mathrm{k})}{\mathrm{Y}_{0}(\beta \mathrm{k})}\right) \\
& \alpha=\rho \beta(\rho>1) \quad \beta \mathrm{k}=\mathrm{x} \text { と和けは } \\
& \frac{\mathrm{J}_{0}(\mathrm{x})}{\mathrm{Y}_{0}(\mathrm{x})}-\frac{\mathrm{Y}_{0}(\rho \mathrm{x})}{\mathrm{J}_{0}(\rho \mathrm{x})}=0
\end{aligned}
$$

(4.8) を解いてて得る。この根は無限に多く存在す るが正根を大さの順に並べてi 番目の根 $x_{V i}$ は

Mc Mahon によれば

$$
\mathrm{x}_{\nu \mathrm{i}}=\delta+\frac{\mathrm{p}}{\delta}+\frac{\mathrm{q}-\mathrm{p}^{2}}{\delta^{3}}+\frac{\mathrm{r}-4 \mathrm{pq}+2 \mathrm{p}^{3}}{\delta^{5}}+
$$

妅に $\delta=\frac{\mathrm{i} \pi}{\rho-1} \quad \mathrm{p}=\frac{\mathrm{m}-1}{8 \rho}$

$$
\left.\begin{array}{c}
\mathrm{q}=\frac{4(\mathrm{~m}-1)(\mathrm{m}-25)\left(\rho^{3}-1\right)}{3(8 \rho)^{3}(\rho-1)} \\
\mathrm{r}=\frac{32(\mathrm{~m}-1)\left(\mathrm{m}^{2}-114 \mathrm{~m}+1073\right)\left(\rho^{5}-1\right)}{5(8 \rho)^{5}(\rho-1)} \\
\mathrm{m}=4 \nu^{2}
\end{array}\right\}
$$

こっで必要なのは最初の根だから $\mathrm{i}=1$ である。

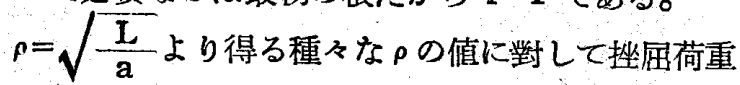
$\mathrm{P}_{\mathrm{cr}}=\frac{\pi^{2} \mathrm{EI}_{3}}{\varepsilon \mathrm{a}^{2}}$ を求めれば $\varepsilon$ の值は次のやうになる。

\begin{tabular}{c|c|c|c|c}
\hline$\rho$ & $\sqrt{5}$ & 2 & $\sqrt{3}$ & $\sqrt{2}$ \\
\hline$\varepsilon$ & 6.1 & 4 & 2.14 & 0.685 \\
\hline
\end{tabular}

\section{$\$ 5 \mathrm{~m}=2$ なる場合の解}

$m=2 \quad n=-2$ の時は $\nu=\infty$ だから Bessel 函數 では求められない。

(2.2) 式は

$$
\begin{aligned}
& \mathrm{x}^{2} \frac{\mathrm{d}^{2} \mathrm{y}}{\mathrm{dx}^{2}}+\mathrm{k}^{2}(\mathrm{y}-\delta)+\mathrm{k}^{2} \theta=0 \\
& \text { 但 } \mathrm{k}=\sqrt{\frac{\mathrm{Pa}^{2}}{\mathrm{El}_{0}}}, \quad \theta=\frac{\mathrm{M}}{\mathrm{P}}
\end{aligned}
$$

この一般解は簡單な力法*により次の如くに表わすこ とが出來る。

$\left.\mathrm{y}=\mathrm{x}^{\frac{1}{2}}\left\{\mathrm{C}_{1} \cos (\beta \log \mathrm{x})+\mathrm{C}_{2} \sin (\beta \log \mathrm{x})\right\}+\delta=\theta\right\}$ 妶に $\beta=\sqrt{k^{2}-\frac{1}{4}} \quad k^{2}-\frac{1}{4}>0$

(5.2)より

$$
\begin{aligned}
& \frac{d y}{d x}=\frac{C_{1}}{2 \sqrt{x}}\{\cos (\beta \log x)-2 \beta \sin (\beta \log x)\} \\
& +\frac{C_{2}}{2 \sqrt{x}}\{(\sin (\beta \log x)+2 \beta \cos (\beta \log x)\}
\end{aligned}
$$

柱脚固定の境界條件は

固定端に於 $\mathrm{x}=\mathrm{a}$ の時 $\mathrm{y}=0, \frac{\mathrm{dy}}{\mathrm{dx}}=0$

上端 $\mathrm{B}, \mathrm{C} K$ 於 $\tau \mathrm{x}=\mathrm{L}$ の時 $\mathrm{y}=\delta, \frac{\mathrm{dy}}{\mathrm{dx}}=\frac{\mathrm{Mb}}{6 \mathrm{EI}}$

（5.4）に依つて $\mathrm{C}_{1}, \mathrm{C}_{2}$ を定める。

$$
\left.\begin{array}{l}
\mathrm{C}_{1}=\frac{(\theta-\delta)\{\sin (\beta \log \mathrm{a})+2 \beta \cos (\beta \log \mathrm{a})\}}{2 \sqrt{\mathrm{a} \beta}} \\
\mathrm{C}_{2}=\frac{-(\theta-\delta)\{\cos (\beta \log \mathrm{a})-2 \beta \sin (\beta \log \mathrm{a})\}}{2 \sqrt{\mathrm{a} \beta}}
\end{array}\right\}
$$

$$
\begin{aligned}
\therefore \mathrm{y}= & \frac{\mathrm{x}^{\frac{1}{2}}(\theta-\delta)}{2 \sqrt{\mathrm{a}} \beta}\left\{\sin \left(\beta \log \frac{\mathrm{a}}{\mathrm{x}}\right)\right. \\
& \left.+2 \beta \cos \left(\beta \log \frac{\mathrm{a}}{\mathrm{x}}\right)\right\}+(\delta-\theta) \\
& \frac{\mathrm{dy}}{\mathrm{dx}}=\frac{(\theta-\delta)\left(1+4 \beta^{2}\right)}{4 \sqrt{\mathrm{ax}} \beta} \sin \left(\beta \log \frac{\mathrm{a}}{\mathrm{x}}\right)
\end{aligned}
$$

こっの丽式に境界條件式 (5.5) を適用すると

$$
\begin{aligned}
& \left\{\sin \left(\beta \log \frac{a}{L}\right)+2 \beta \cos \left(\beta \log \frac{a}{L}\right)\right. \\
& \left.-2 \sqrt{\frac{a}{L}} \beta\right\} \theta-\left\{\sin \left(\beta \log \frac{a}{L}\right)\right. \\
& \left.+2 \beta \cos \left(\beta \log \frac{a}{L}\right)\right\}_{\delta}=0 \\
& \left\{\left(1+4 \beta^{2}\right) \sin \left(\beta \log \frac{a}{L}\right)-4 \omega \sqrt{a L} \beta\right\} \theta \\
& -\left\{\left(1+4 \beta^{2}\right) \sin \left(\beta \log \frac{a}{L}\right)\right\}_{\delta=0}
\end{aligned}
$$

$\theta, \delta$ は同时に零となることはない。依つて $\theta, \delta$ 倸 數にて作れる行列式 $\Delta$ ば零である。 $\Delta=0$ この式を展開して

$$
\tan \left(\beta \log \frac{\mathrm{a}}{\mathrm{L}}\right)=\frac{4 \beta \omega}{1-2 \omega \mathrm{L}+4 \beta^{2}}
$$

* Forsyth: Treatise on Differential Equation 策6 版 (1928) $\S 47$ p82 悲 


$$
=\frac{2 \sqrt{k^{2}-\frac{1}{4}}}{12 a s-L}
$$

茲に $\omega=\frac{\mathrm{Pb}}{6 \mathrm{EI}_{1}}=\frac{\mathrm{k}^{2}}{6 \mathrm{a}^{2} \mathrm{~s}}, \mathrm{~s}=\frac{\mathrm{aI}_{1}}{\mathrm{bI}_{0}}$

（5.7）が挫屈荷重を定める式である。

$$
\begin{aligned}
& I_{1} \rightarrow \infty \text { とすれは } \\
& \tan \left(\beta \log \frac{a}{L}\right)=0 \quad \therefore \beta \log \frac{a}{L}=\pi \\
& \therefore P_{c r}=\left(\frac{1}{4}+\frac{\pi^{2}}{c^{2}}\right) \frac{E I_{0}}{a^{2}} \\
& \left(c=\log \frac{a}{L}\right)
\end{aligned}
$$

(5.8) は兩端固定の長柱の挫屈荷重である。

(5.7)より得る最小根(圖解により求める)を $\xi_{1}$ と すれば

$$
\begin{aligned}
& \mathrm{P}_{\mathrm{cr}}=\xi_{1}{ }^{2}\left(\frac{1}{4}+\frac{\xi_{1}^{2}}{\mathrm{c}^{2}}\right) \frac{\mathrm{EI}_{0}}{\mathrm{a}^{2}} \\
&\left(\mathrm{c}=\log \frac{\mathrm{a}}{\mathrm{L}}\right)
\end{aligned}
$$

\section{$\$ 6 \mathrm{~m}=3$ なる場合の解}

$$
\begin{aligned}
& \mathrm{m}=3, \mathrm{n}=-3 \therefore \nu=-1 \mathrm{z}=-2 \mathrm{kx}^{-\frac{1}{2}} \\
& \mathrm{z}_{\mathrm{L}}=\alpha \mathrm{k} \mathrm{z}_{\mathrm{a}}=\beta \mathrm{k} \alpha=-\frac{2}{\sqrt{\mathrm{L}}} \beta=-\frac{2}{\sqrt{\mathrm{a}}}
\end{aligned}
$$

(2.15) は

$$
\begin{aligned}
\varphi & =\mathrm{J}_{-1}(\alpha \mathrm{k}) \mathrm{Y}_{-1}(\beta \mathrm{k})-\mathrm{J}_{-1}(\beta \mathrm{k}) \mathrm{Y}_{-1}(\beta \mathrm{k}), \\
& =\mathrm{J}_{1}(\alpha \mathrm{k}) \mathrm{Y}_{1}(\beta \mathrm{k})-\mathrm{J}_{1}(\beta \mathrm{k}) \mathrm{Y}_{1}(\alpha \mathrm{k}), \\
\xi & =\mathrm{J}_{1}(\beta \mathrm{k}) \mathrm{Y}_{0}(\alpha \mathrm{k})-\mathrm{Y}_{1}(\beta \mathrm{k}) \mathrm{J}_{0}(\alpha \mathrm{k}) \\
\eta & =\mathrm{J}_{0}(\alpha \mathrm{k}) \mathrm{Y}_{0}(\beta \mathrm{k})-\mathrm{J}_{0}(\beta \mathrm{k}) \mathrm{Y}_{0}(\alpha \mathrm{k}) \\
\psi & =\mathrm{Y}_{1}(\alpha \mathrm{k}) \mathrm{J}_{0}(\beta \mathrm{k})-\mathrm{J}_{1}(\alpha \mathrm{k}) \mathrm{Y}_{0}(\beta \mathrm{k})
\end{aligned}
$$

之等を (2.14) に代入して簡單にすると

$$
\frac{\mathrm{pY}_{2}(\alpha \mathrm{k})-\mathrm{J}_{2}(\alpha \mathrm{k})}{\mathrm{pY}_{1}(\alpha \mathrm{k})-\mathrm{J}_{1}(\alpha \mathrm{k})}=\frac{8 \omega}{\alpha^{3} \mathrm{k}}
$$$$
\text { 但 } \mathrm{p}=\frac{\mathrm{J}_{2}(\beta \mathrm{k})}{\mathrm{Y}_{2}(\beta \mathrm{k})}
$$

$\alpha=\frac{-2}{\sqrt{L}} \quad \beta=\frac{-2}{\sqrt{a}}$ 厄゙るから次の事に注意 ナる。

$\mathrm{J}_{v}(-\mathrm{z})=(-1)^{\vee} \mathrm{J}_{\nu}(\mathrm{z})$ でむり第二種の Bessel 函 數はレ が整數なる㭙は第 1 報* (1.9) 式に於てレ =0, 1 に對しては

$$
\left.\begin{array}{l}
\left.\mathrm{Y}_{1}(-z)=-\mathrm{Y}_{1}(z)+2(2 \nu+1) \mathrm{iJ}_{1}(z)\right)_{1}=\sqrt{-1} \\
\mathrm{Y}_{2}(-z)=\mathrm{Y}_{2}(z)+2(2 \nu+1) \mathrm{iJ}_{2}(z)
\end{array}\right\}
$$

虚數部を捨てれば (6.1)は

$$
\begin{aligned}
& \frac{\mathrm{pY}_{0}(\alpha \mathrm{k})-\mathrm{J}_{\mathrm{g}}(\alpha \mathrm{k})}{-\mathrm{pY}_{\mathrm{I}}(\alpha \mathrm{k})+\mathrm{J}_{1}(\alpha \mathrm{k})}=-\frac{8 \omega}{\alpha^{3} \mathrm{k}} \\
& =-\frac{4 \mathrm{k}}{3 \mathrm{a}^{2} \mathrm{~s} \alpha^{3}}
\end{aligned}
$$

但

$$
\begin{gathered}
\alpha=\frac{2}{\sqrt{\mathrm{L}}} \quad \beta=\frac{2}{\sqrt{\mathrm{a}}} \quad \mathrm{p}=\frac{\mathrm{J}_{2}(\beta \mathrm{k})}{\mathrm{Y}_{2}(\beta \mathrm{k})} \\
\omega=\frac{\mathrm{Pb}}{6 \mathrm{EI}_{1}}=\frac{\mathrm{k}^{2}}{6 \mathrm{a}^{2} \mathrm{~s}} \quad \mathrm{~s}=\frac{\mathrm{aI}_{1}}{\mathrm{bI}_{0}} \cdots \cdots(6 .)
\end{gathered}
$$

(6.2)を圖解するため右邊を變形する。

$$
\begin{aligned}
& -\frac{8 \omega}{\alpha^{3} \mathrm{k}}=-\alpha \mathrm{k} \times \frac{8 \omega}{\alpha^{4} \mathrm{k}^{2}}=-\alpha \mathrm{k} \frac{8 \mathrm{~Pb}}{6 \mathrm{EI}} / \frac{16}{\mathrm{~L}^{2}} \\
& \times \frac{\mathrm{Pa}^{3}}{\mathrm{EI}_{0}}=-\alpha \mathrm{k} \frac{\mathrm{L}^{2} \mathrm{bI}_{0}}{12 \mathrm{a}^{3} \mathrm{I}_{1}}
\end{aligned}
$$

$\alpha=\rho \beta(\rho<1) \beta k=\xi$ と报くと

$$
\frac{\frac{\mathrm{J}_{2}(\xi)}{\mathrm{Y}_{2}(\xi)} \mathrm{Y}_{2}(\rho \xi)-\mathrm{J}_{0}(\rho \xi)}{\rho \xi\left\{\frac{\mathrm{J}_{2}(\xi)}{\mathrm{Y}_{2}(\xi)} \mathrm{Y}_{1}(\rho \xi)-\mathrm{J}_{1}(\rho \xi)\right\}}=\frac{\mathrm{L}^{2} \mathrm{bI}_{0}}{12 \mathrm{a}^{3} \mathrm{I}_{1}}
$$

この式の左邊を ค 老媒介變數とする $\xi$ の函數として 圖表をかけばよい。圆から求めた根を 乡とすれば

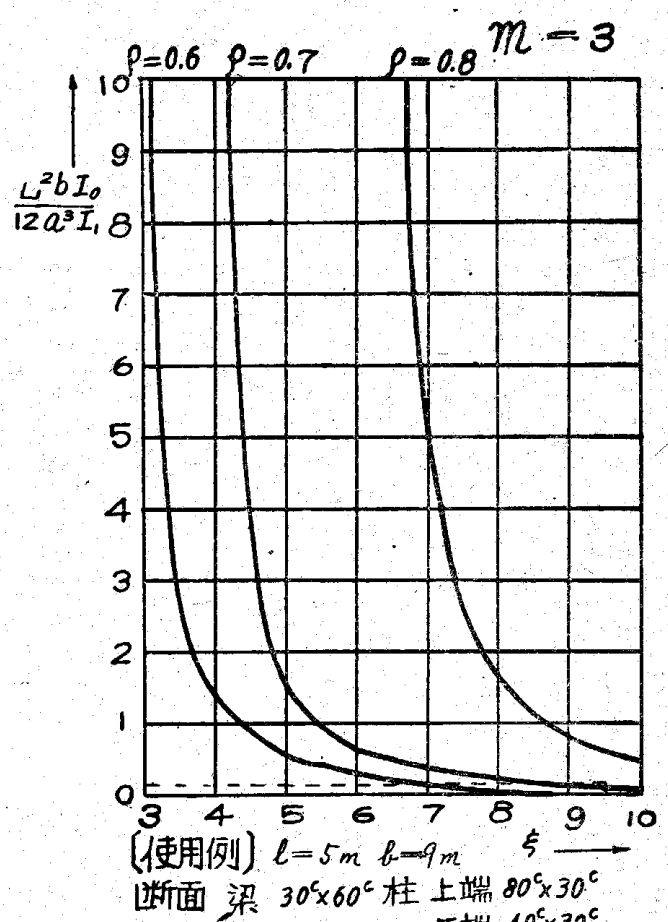

$$
\begin{aligned}
& \rho=\sqrt{\frac{5}{10}}=0.7 \quad \frac{L^{2} b I_{0}}{12 a^{3} I_{i}}=\frac{10^{2} \times 90^{c}}{12 \times 5 \times 0.3 \times 0.4^{3}} \\
& \therefore \xi_{1}=9.5 \\
& \therefore P_{c r}=\xi_{1}^{2} \frac{L E_{c} I_{0}}{4 a^{3}}=9.5^{2} \times \frac{10 \times 2.1 \times 10^{4} \times 0.3 \times 0.4^{3}}{4 \times 5^{3} \times 12} \\
& =60.5 \mathrm{tm}
\end{aligned}
$$

第 3 圖 


$$
\mathrm{P}_{\mathrm{cr}}=\xi_{1}^{2} \frac{\mathrm{LEI}_{0}}{4 \mathrm{a}^{3}}
$$

$\$ 7 \mathrm{~m}=4$ なる場合の解

$\mathrm{m}=4, \mathrm{n}=-4$ の時 $\mathrm{\nu}=-\frac{1}{2}, \mathrm{z}=-\frac{\mathrm{k}}{\mathrm{x}}$, $z_{L}=-\frac{k}{L} \quad z_{a}=-\frac{k}{a}$

レが半奇數なる時は (3.1) の關係がある。

之を(2.15) に代入し，更にその結果を (2.14) に 入れると

$$
\begin{aligned}
& \tan \frac{\mathrm{kl}}{\mathrm{aL}}=\frac{\mathrm{k}\left(\omega \mathrm{L}^{2}-\mathrm{L}+\mathrm{a}\right)}{\mathrm{a} \omega \mathrm{L}^{2}-\mathrm{aL}-\mathrm{k}^{2}} \\
& =\frac{\mathrm{k}\left(\mathrm{L}^{2} \mathrm{k}^{2}+6 s \mathrm{a}^{4}-6 s \mathrm{~s}^{3} \mathrm{~L}\right)}{a \mathrm{~L}^{2} \mathrm{k}^{2}-6 \mathrm{sa}^{3} \mathrm{k}^{2}-6 s \mathrm{~s}^{4} \mathrm{~L}} \\
& \text { 妶 } \mathrm{s}=\frac{a I_{1}}{\mathrm{bI}_{0}} \\
& \mathrm{I}_{1 \rightarrow \infty}
\end{aligned}
$$

$$
\tan \frac{\mathrm{kl}}{\mathrm{aL}}=0 \therefore \frac{\mathrm{kl}}{\mathrm{aL}}=\pi \therefore \mathrm{P}_{\mathrm{cr}}=\frac{\pi^{2} \mathrm{~L}^{2} \mathrm{EI}_{0}}{\mathrm{a}^{2} 1^{2}}
$$

これは圓形页は角柱の尖細りの挫屈荷重となる。 (7.1)を圖解で解いた最小根を $\xi_{1}$ とすれば

$$
\mathrm{P}_{\mathrm{cr}}=\xi_{1}{ }^{2} \frac{\mathrm{L}^{2} \mathrm{EI}_{0}}{\mathrm{a}^{2} 1^{2}}
$$

これが求める挫屈荷重である。

\section{$\$ 8$ 結 語}

以上を要するに柱脚固定の變斷面部材の門型ラーメ ンに就て挫屈荷重を求めたのであるが更に斷面の變化 をラチス材を考慮しで $\mathrm{I}(\mathrm{x})=\mathrm{I}_{0}\left(\frac{\mathrm{x}}{\mathrm{a}}\right)^{2}+\nu \mathrm{I}_{0}\left(\frac{\mathrm{x}}{\mathrm{a}}\right)^{3}$ そ假定せる場合に就て研究せるるのを報告する豫定で ある。

* 蛙田捨太郎：變斷面の部材を有する架構の解法に就て (建築學會研究報告第 1 號 昭和 24 年 5 月參照)

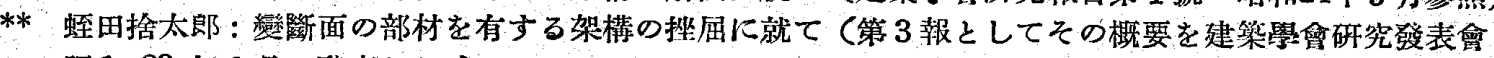
昭和 23 年 8 月に發表した。) 